\title{
Giant coronary aneurysm in a woman with Sjogren's syndrome and rheumatoid arthritis history: case report
}

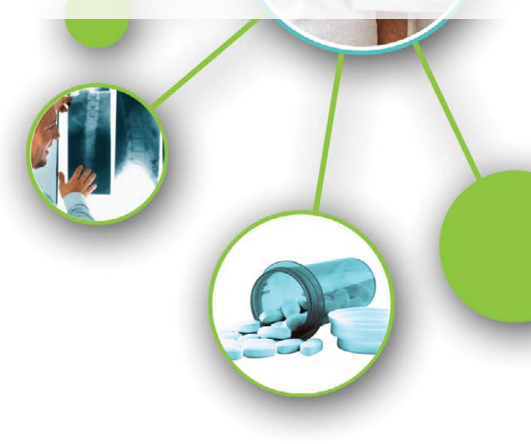

Coronary artery aneurysm (CAA) is defined as a focal or diffuse dilatation of a coronary artery, greater than 1.5 times the diameter of normal adjacent segments. Here we reported a 58-year-old woman with a background of rheumatoid arthritis and Sjogren syndrome history. Coronary angiography and Computerised tomographic (CT) coronary angiography confirmed a giant coronary aneurysm (maximum diameters of aneurysm were $12.6 \mathrm{~mm}$ and $8.8 \mathrm{~mm}$ ) in the branch of left anterior descending artery, might with a fistulous communication with the pulmonary artery. To the best of our known, there were no reports about giant CCA in patient with the rheumatoid arthritis and Sjogren's syndrome history.

\section{KEYWORDS: coronary artery aneurysm, Sjogren's syndrome, rheumatoid arthritis}

\section{Introduction}

Coronary artery aneurysm (CAA) is defined as a focal or diffuse dilatation of a coronary artery, greater than 1.5 times the diameter of normal adjacent segments. And the Committee of the American Heart Association has defined giant aneurysms as those $>8 \mathrm{~mm}[1,2]$. The incidence giant coronary artery aneurysm is hard to be determined, because few studies have been reported in literature. Other literature showed that the incidence of coronary artery aneurysm in the general population range from $0.02 \%$ to $0.04 \%$ and the incidence range from $1.1 \%$ to $5 \%$ in patients undergoing coronary angiopathy [3]. However the giant coronary aneurysms are rarely reported. The most affected coronary artery is the right coronary artery RCA, followed by the left anterior descending artery LAD and left circumflex ( LCX) [3,4].

CAA is usually asymptomatic, so always identified during coronary angiography incidentally. Patient could have complications when CAA rupture, thrombosis, embolization, dissection, mechanical obstruction, and erosion into surrounding structures with or without fistula formation [3-5]. The complications are main ischemic symptoms, such as angina, myocardial infarction, and with supraventricular or malignant ventricular tachyarrhythmia [5]. Here we reported a case of giant coronary aneurysm in a 58-year-old woman with a background of rheumatoid arthritis and Sjogren syndrome history.

\section{Case report}

A 58-year-old woman with a background of rheumatoid arthritis and Sjogren Syndrome history was referred to our hospital because chest pain once. She has one history of anginalike chest pain 15 days before admitted, which continue about 10 minutes. She also had medical history of rheumatoid arthritis 1 years and Sjogren Syndrome about 10 years. Methotrexate, hydroxychloroquine and prednisone were taken orally to control both diseases. She had not the risk factors for coronary artery diseases (hypertension, obesity, diabetes and smoking). On physical examination, her blood pressure was $130 / 70 \mathrm{mmHg}$ and pulse rate was $80 /$ min. Physical examination results were normal. Her ECG, Cardiac enzyme levels, Computed tomography (CT) of chest and abdomen were normal. Both transthoracic echocardiogram and artery echocardiogram were also normal.

Coronary angiography (FIGURE 1) revealed demonstrated a giant aneurysm in the branch of LAD, which might be the sinus tract to the pulmonary artery. When we performed coronary angiography, a turbulent flow was found within the CAA and contrast agent retention in the CAA. Only a 20\% stenosis was found in the middle of LAD, The LCX, LM, RCA were normal, but RCA was thin and small. Computerized tomographic (CT) coronary angiography (FIGURE 2) and three-dimensional reconstruction of CT images (FIGURE 3) further characterized the
Shenggang $\mathrm{Zhao}^{1}, \mathrm{LiXu}^{2}$, Jianjiang $X u^{1}$, ZhangBin ${ }^{1}$, Jianqin Zhang ${ }^{1} \&$

Liqin Jiang ${ }^{* 1}$

${ }^{1}$ Department of Cardiology, The Second Affiliated Hospital of Jiaxing Unicersity, Jiaxing, ZheJiang Province, China

${ }^{2}$ Department of Radiology, The Second Affiliated Hospital of Jiaxing Unicersity, Jiaxing, ZheJiang Province, China

*Author for correspondence:

jiangliqin@medmail.com.cn 


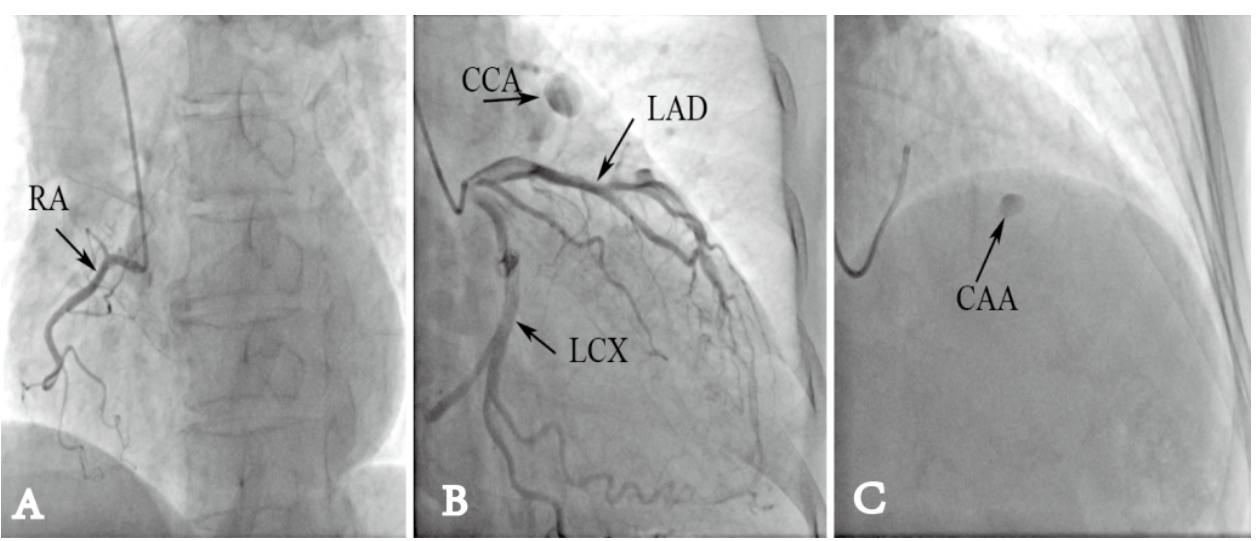

FIGURE 1. Coronary angiography showed RCA was thin and small with no stenosis ( $A$ ) a giant aneurysm in the branch of LAD, which might be the sinus tract to the pulmonary artery (B) Contrast agent retention in the CAA (C) The LAD , LCX and LM were normal.

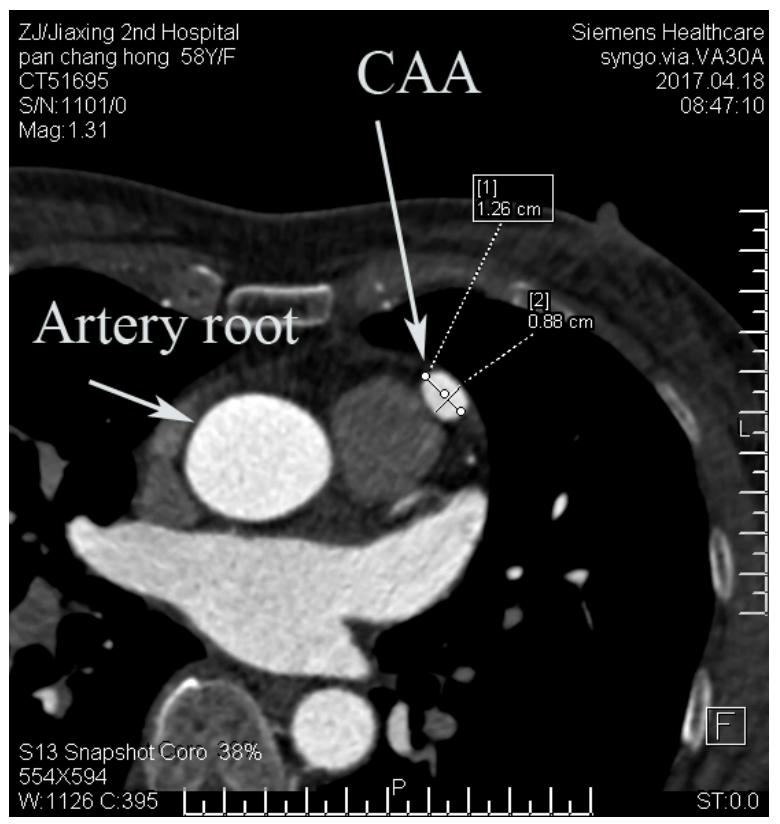

FIGURE 2. Computerized tomographic (CT) coronary angiography revealed the maximum diameters of aneurysm were $12.6 \mathrm{~mm}$ and $8.8 \mathrm{~mm}$.
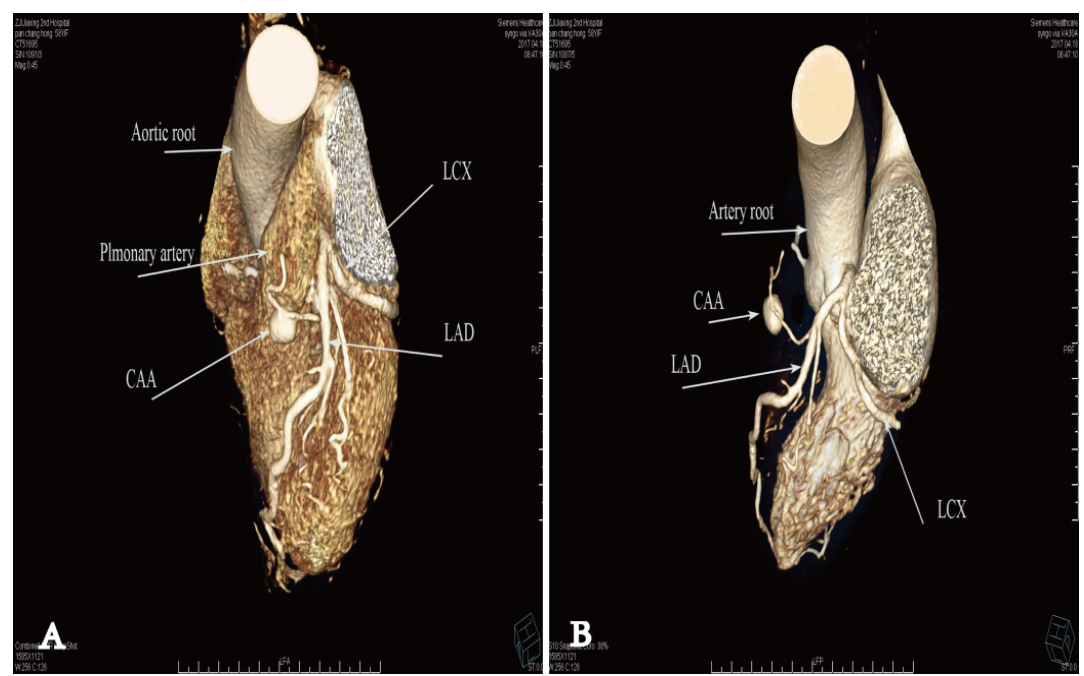

FIGURE 3. Three-dimensional reconstruction of CT images confirmed that the giant coronary aneurysm arising from the branch of LAD. 
aneurysmal to be arising from the branch of LAD might with fistulous connection to the pulmonary artery.

\section{Discussion}

The precise path mechanism of CAA remains a controversial topic of discussion. But currently the reasons could be divided into congenital or mainly acquired according to literature. The congenital CAA is generally found in young patients and are linked to Kawasaki disease and Marfan syndrome. The main acquired etiological factor for the development of CAA is atherosclerotic coronary artery disease, which is present in $50 \%$ to $52 \%$ of cases $[2,6,7]$. Apart from atherosclerosis, the other etiologies of CAAs are systemic lupus erythematosus, trauma, mycotic embolism, cocaine use, complicated percutaneous coronary interventions $[1,2]$. In our case, we thought rheumatoid arthritis and Sjogren's Syndrome are the main cause of huge CAA because the patient had no risk of atherosclerosis except for age and ECG, transthoracic echocardiogram and artery echo were also normal. Perhaps atherosclerosis factors paly a small role with Sjogren's Syndrome and rheumatoid arthritis together contribute to giant CAA, because only a $20 \%$ stenosis was found in the middle of LAD. The LCX, LM, RCA were normal revealed by Coronary angiography and Computerized tomographic (CT) coronary angiography.

There is no firmly establish guidelines and evidence-based consensus on the optimal management strategy of giant coronary artery aneurysms because rarity of giant coronary aneurysms and management varies based on the underlying etiology, size and location of the aneurysm, clinical symptoms, and associated comorbidities. Therefore, it is recommended that patients be managed individually. Currently treatments options of CAA include three as follow: conservative medical management with antiplatelet drugs or anticoagulant drugs; percutaneous intervention with covered stents; and surgical excision of the CAA accompanied by distal bypass surgery $[4,8,9]$.

For small, asymptomatic coronary artery aneurysms patients without severe CAD, conservative medical management with antiplatelet therapy and addition of anticoagulant drugs is recommended to reduce thromboembolic complications. Percutaneous intervention with covered stents may be concerned in eligible symptomatic patients. Surgery has been recommended for all CAA patients when have risk of thrombosis or rupture, particularly for giant aneurysm. Aneurysms compromising the superior cava vein, the right atrium or even the right ventricular inflow tract are absolute indications for surgery $[4,8,9]$. But other experts insist that CAAs bear a significant risk of severe complications and have a high risk of mortality. A more aggressive surgical approach should be recommended [2,3].

\section{Acknowledgement}

The present study was supported by the Science technology department of JiaXing(2016AY23056). Traditional Chinese medicine science and technology of ZheJiang province (2015ZA204) 


\section{REFERENCES}

Dajani AS, Taubert KA, Takahashi $\mathrm{M}$, et al. Guidelines for long-term management of patients with Kawasaki disease. Report from the Committee on Rheumatic Fever, Endocarditis, and Kawasaki Disease, Council on Cardiovascular Disease in the Young, American Heart Association. Circulation 89, 916-922 (1994).

Wang H, Zhang Y, Xie Y, Wang H, Yuan J. Giant right coronary artery aneurysms presenting as a cardiac mass: Case report. Medicine 95,e4924 (2016).

Keyser A, Hilker MK, Husser O, Diez C, Schmid C. Giant coronary aneurysms exceeding $5 \mathrm{~cm}$ in size. Interact. Cardiovasc. Thorac. Surg. 15, 33-36 (2012).

Tuncer E, Onsel Turk U, Alioglu E. Giant saccular aneurysm of the left main coronary artery. J. Geriatr. Cardiol. 10, 110-112 (2013).

Lee CH, Son CW, Park JS. Giant left anterior descending artery aneurysm resulting in sudden death. Hellenic. J. Cardiol. 57,263-266 (2016).

Yanik A, Arslan U, Akcay M, Menekse S, Yazgan UG. Giant Coronary Artery Aneurysm Causing Acute Anterior Myocardial Infarction. Case Rep. Med. 5180472 (2016).

Yoshihiro MKY, Yuichi I, Hiroki U, et al. Acute Myocardial Infarction That Resulted From Poor Adherence to Medical Treatment for Giant Coronary Aneurysm The Importance of Patient Education in the Chronic Phase of Kawasaki Disease. Int. Heart J. 56, 551-554 (2015).

Barr J, Acharya MN, Kourliouros A, Raja SG. Technical Considerations of Giant Right Coronary Artery Aneurysm Exclusion. Case Rep. Surg. 3795640 (2016).

Siregar S, Hoseyni GS, Herwerden LA. Covered stents in giant coronary artery aneurysm. Eur. Heart J. 31,2823 (2010). 